\title{
Mid-Term Radiological and Functional Outcomes of Bicondylar Tibial Plateau Fractures Managed with Open Reduction and Internal Fixation Using Dual Plates
}

"Angelo V. Vasiliadis, ${ }^{1,2}$ Frideriki Poutoglidou, ${ }^{1}$ Dimitrios Metaxiotis, ${ }^{1}$ Anastasios Mpeletsiotis ${ }^{1}$

\begin{abstract}
Objectives: This study aimed to evaluate the mid-term radiological and functional outcome of tibial plateau fractures treated by plating. Methods: This study was conducted at the General Hospital of Thessaloniki 'Papageorgiou', Thessaloniki, Greece. Patients with Schatzker type V and VI tibial plateau fractures who were managed with open reduction and internal fixation using dual plates between January 2011 and December 2018 were included in this retrospective study. The functional evaluation of the patients was carried out with the visual analogue scale (VAS), the health-related quality of life status was measured using the Short Form-36 and the dimensions of pain, stiffness and function were assessed using the Western Ontario and McMaster Universities Arthritis Index. For the radiological outcome evaluation, the modified Rasmussen criteria were used. Results: Fifty-seven patients (30 male and 27 female) were included in the study with a mean follow-up of 50.88 months. There were 23 Schatzker type V and 34 type VI fractures. The majority of patients (86\%) had a good to excellent radiological outcome. The mean VAS score was 1.65 for all the patients. The functional outcome was excellent in the majority of the patients. Among them, 5.3\% $(n=3)$ suffered wound infection and all wounds healed after different treatments. All patients returned to their pre-injury activities of daily living and employment status, while $53 \%$ of the patients returned to sporting activities. Conclusion: The findings support previous literature which has demonstrated that bicondylar tibial plateau fractures can provide good-to-excellent radiological and functional outcomes if they are treated with open reduction and internal fixation with dual plating.
\end{abstract}

Keywords: Tibial; Fracture; Classification; Surgical Procedure; Plating.

\begin{abstract}
AdVANCES IN KNOWLEDGE
The findings of the present study show that bicondylar tibial plateau fractures treated operatively with open reduction and internal fixation (ORIF) with dual plates had good-to-excellent mid-term radiological (modified Rasmussen criteria) and functional (Short Form-36 and Western Ontario and McMaster Universities Arthritis Index) outcomes at mid-term follow-up.

There were no statistically significant differences between the early ( $\leq 48$ hours) and delayed time to surgery with respect to superficial and deep wound infection.

\section{Application to Patient Care}

Early surgical intervention for bicondylar tibial plateau fractures may be the key to successful treatment and improved functional outcomes.
\end{abstract}

\section{$\mathrm{T}$} Ibial plateau fractures COMPrise approximately $2 \%$ of all fractures and tend to have a bimodal age distribution. Males in the fourth decade of their life are more likely to sustain these types of injuries due to high-energy trauma. Meanwhile, tibial plateau fractures are usually a result of lowenergy trauma in females around the age of $70 .{ }^{1}$ These injuries are often associated with soft tissue damage such as vasculature, nerves, ligaments and meniscus. ${ }^{2}$ Schatzker proposed a classification system based on the two-dimensional radiological features, dividing tibial plateau fractures into six types, from type I to VI. ${ }^{3}$ As a supplement to the Schatzker Classification, in 2010, Luo et al. introduced the three-column classification based on computed tomography scan images and three-dimensional reconstruction, thereby increasing the ability to identify the posteromedial fragment. ${ }^{4}$ These types of fractures, which pose a challenge even to a senior orthopaedic surgeon, still yield unsatisfactory results despite appropriate surgical treatment due to their complexity and the associated soft tissue disruption. ${ }^{2,3}$

The objective of tibial plateau operative treatment is to obtain a stable osteosynthesis and to enable early mobilisation of the knee joint. Various techniques have been proposed over the last few decades, each one with its own merits and demerits. Open reduction and internal fixation (ORIF) with various types of plates and screws remains the gold standard. Alternatively, circular external fixation with limited internal 
fixation (hybrid fixator) and arthroscopically assisted percutaneous reduction and internal fixation have been reported for the treatment of tibial plateau fractures..$^{5-7}$ Despite the plethora of techniques, malalignment and secondary osteoarthritis are common complications encountered after surgery for complex tibial plateau fractures. This study aimed to evaluate the mid-term radiological and functional outcome of tibial plateau fractures managed with ORIF using dual plates.

\section{Methods}

This retrospective study was conducted at the General Hospital of Thessaloniki 'Papageorgiou', Thessaloniki, Greece. All patients admitted with the diagnosis of bicondylar tibial plateau fractures over a period of eight years, from January 2011 to December 2018, were included in the study. Only patients with Schatzker type $\mathrm{V}$ and VI managed with dual plating were included. The data were extracted from hospital records and outpatient department notes. The exclusion criteria were: 1) patients with bilateral fractures; 2) patients with ipsilateral femoral fractures (floating knee) or other lower limb fractures; 3 ) open tibial fractures; and 4) severe head injury with neurological deficits.

The fractures were graded preoperatively using Schatzker's classification system of tibial plateau fractures by routine anterior posterior and lateral radiographs of the knee. A computed tomography and a threedimensional reconstruction was also performed to evaluate the fractures' morphology and severity according to the three-column classification [Figure 1]. ${ }^{4}$ According to this classification, patients were categorised as: i) lateral and medial column; ii) lateral and posterior column; iii) medial and posterior column; and iv) lateral, medial and posterior column. A magnetic resonance imaging (MRI) was performed on each patient in order to determine ligament and meniscal injuries. All patients had their limb elevated and immobilised with an above knee cast. If oedema was present and development of haemorrhagic fracture blisters occurred, surgery was delayed until soft tissue recovery, as evidenced by reduction of the swelling and appearance of skin lines and 'wrinkle' signs.

All patients underwent ORIF of the fracture through an anterolateral approach for fixation of the lateral column, an anteromedial approach for fixation of the medial column and a posteromedial approach for fixation of the posteromedial column. No temporary knee-spanning external fixation or bony skeletal traction was required preoperatively. The fractures were manipulated and fixed with plates under fluoroscopic guidance in order to assist and assess the reduction. The metaphyseal void was filled with allogenic bone grafts to support reduction and to promote fracture healing in depressed and comminuted fractures patterns.

All patients in this study were contacted and a last follow-up evaluation was scheduled, during which radiographs of the knee were obtained in the anteroposterior and lateral views to assess the articular depression, the condylar widening, the varus/valgus angulation and any evidence of posttraumatic degenerative changes [Figure 2]. Regarding functional outcome, the pain was assessed using the visual analogue scale (VAS), the health-related quality of life status was measured using the Short Form36 (SF-36) and the dimensions of pain, stiffness and function were assessed using the Western Ontario and McMaster Universities Arthritis Index (WOMAC). The radiological outcome was assessed using modified Rasmussen criteria for radiological assessment. Range of motion (ROM) was measured using a standard goniometer. Finally, all complications were documented during the last follow-up.

The statistical analysis was conducted with Jamovi software version 1.1.9. ${ }^{8}$ Continuous variables were given as mean and standard deviation, while nominal variables were expressed as number of cases. The Shapiro-Wilk test was used to determine whether data were normally distributed. Continuous variables were analysed using the independent samples t-test or the Mann-Whitney U-test. Categorical variables were analysed using Pearson's Chi-square test or Fisher's exact test. A $P$-value of $<0.05$ was considered to be statistically significant.

This study was examined and approved by the Institutional Review Board at the General Hospital of Thessaloniki 'Papageorgiou', Thessaloniki, Greece (56805.2020). Informed consent for medical photographs was obtained from the patients.

\section{Results}

A total of 57 patients were included in the study. All patients in the present study underwent surgery at a mean interval of 3.09 days (range $=1-9$ days). The mean duration of hospitalisation was 8.19 days ranging from 6-13 days. A follow-up ranging from 26-78 months (mean $=50.88 \pm 16.56$ months) showed that all fractures were healed [Table 1]. At the final follow-up, none of the patients reported knee instability while the mean ROM was $119.35 \pm 4.84$ degrees (range $=110-$ 125 degrees) for type $\mathrm{V}$ and $110 \pm 10.3$ degrees (range = 90-125 degrees) for type VI fractures [Table 2]. reatment complications were rare, involving two cases of superficial wound infection (one male and one 
Table 1: Patients' characteristics of Schatzker Type V and VI tibial plateau fracture $(\mathrm{N}=57)$

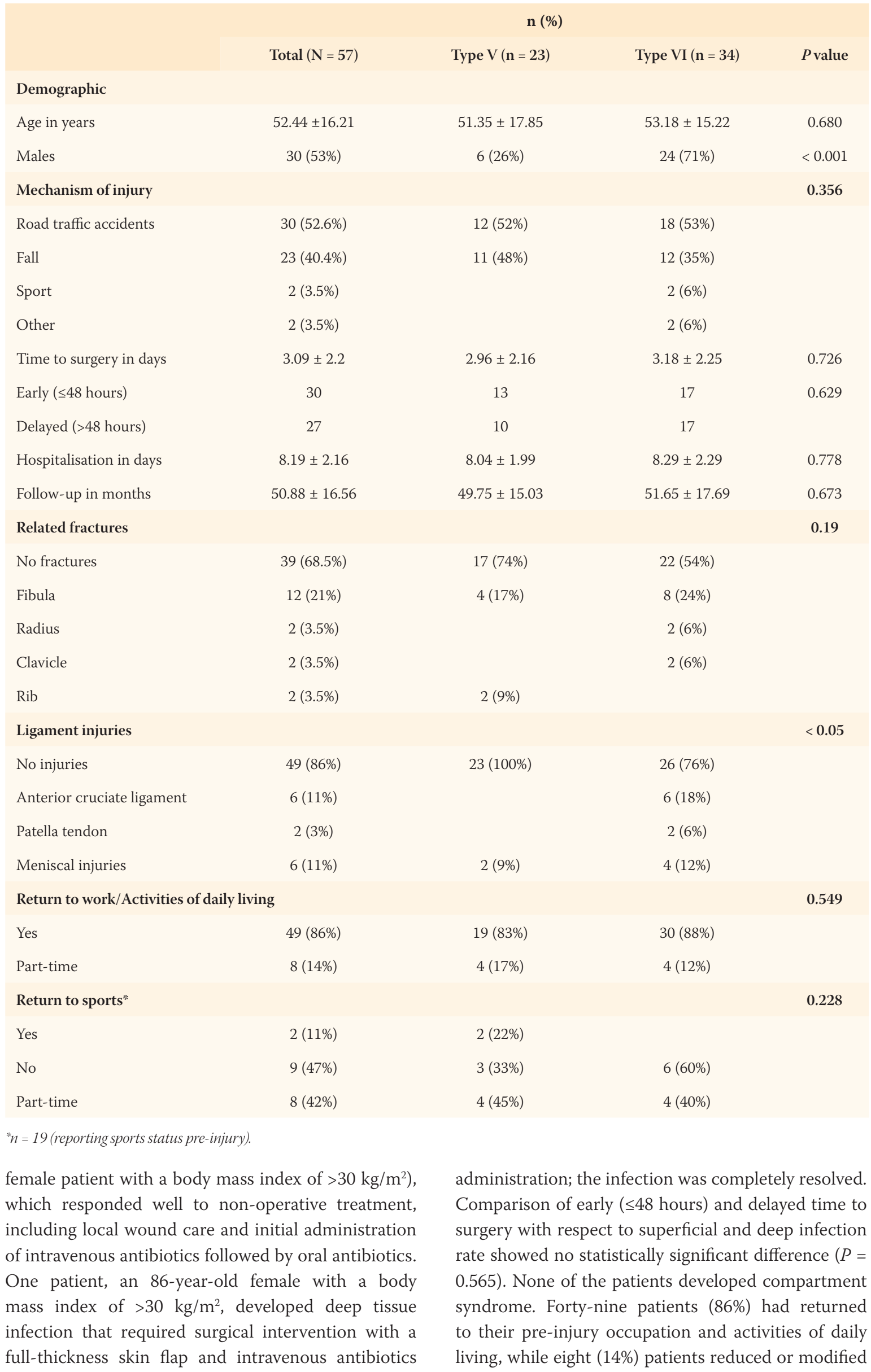


Table 2: Functional and radiological outcomes of tibial plateau fractures $(\mathrm{N}=57)$

\begin{tabular}{|c|c|c|c|c|}
\hline & \multirow[t]{2}{*}{ Total $(\mathrm{n}=57)$} & \multicolumn{2}{|c|}{ Schatzker Classification } & \multirow[t]{2}{*}{$P$ value } \\
\hline & & Type V (n = 23) & Type VI $(n=34)$ & \\
\hline VAS & $1.65 \pm 0.67$ & $1.39 \pm 0.66$ & $1.82 \pm 0.63$ & $<0.05$ \\
\hline SF-36 & $88.68 \pm 4.48$ & $90.65 \pm 4.07$ & $87.35 \pm 4.31$ & $<0.05$ \\
\hline WOMAC & $23.80 \pm 6.28$ & $21.64 \pm 7.14$ & $25.26 \pm 5.24$ & 0.108 \\
\hline Rasmussen* & & & & 0.865 \\
\hline Excellent & 14 & 6 & 8 & \\
\hline Good & 35 & 14 & 21 & \\
\hline Fair & 7 & 3 & 4 & \\
\hline Poor & 1 & & 1 & \\
\hline ROM in degrees & $113.77 \pm 9.65$ & $119.35 \pm 4.84$ & $110 \pm 10.3$ & $<0.001$ \\
\hline
\end{tabular}

VAS = visual analogue scale; SF-36 = Short-Form 36 Health Survey; WOMAC = Western Ontario and McMaster Universities Arthritis Index; ROM = range of motion.

*Rasmussen assessment criteria for radiological outcome.
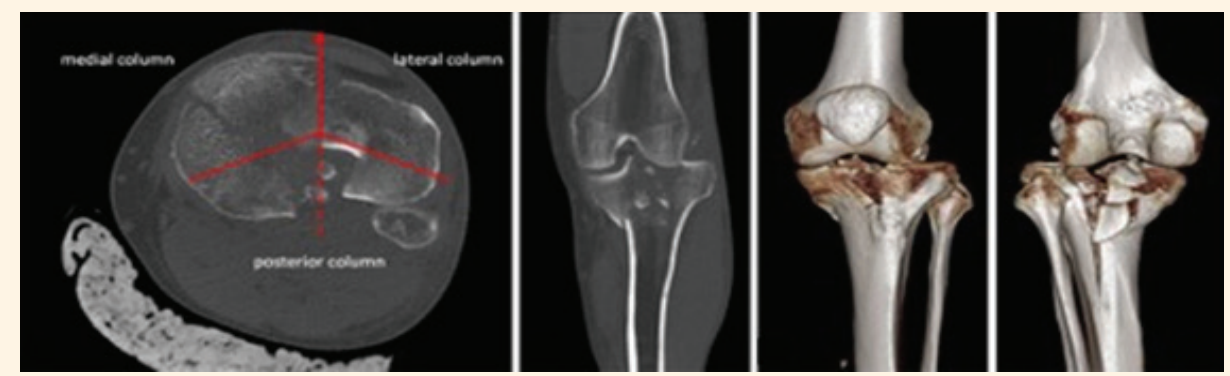

Figure 1: All the fractures were classified preoperatively with the three-column classification system using twodimensional and three-dimensional (3D) computed tomography images.

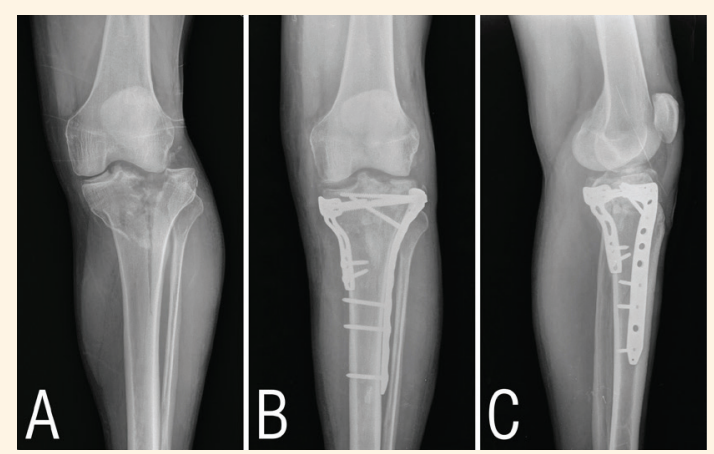

Figure 2: Plain radiograph (A) of left tibia showing the bicondylar tibial fracture and metaphyseal involvement (Schatzker Type VI). Plain radiographs (B, C) taken at the final follow-up showing the anatomic reduction.

their working status. Out of 57 patients, 19 reported a sport status before injury, while approximately $67 \%$ and $40 \%$ returned to sport activity after surgical treatment for Schatzker type V and type VI fractures, respectively [Table 1].

At the final follow-up, the mean VAS score was 1.39 (range $=0-2)$ for type $\mathrm{V}$ and $1.82($ range $=1-3$ ) for type VI fractures, the SF-36 was $90.65 \pm 4.07$ (range

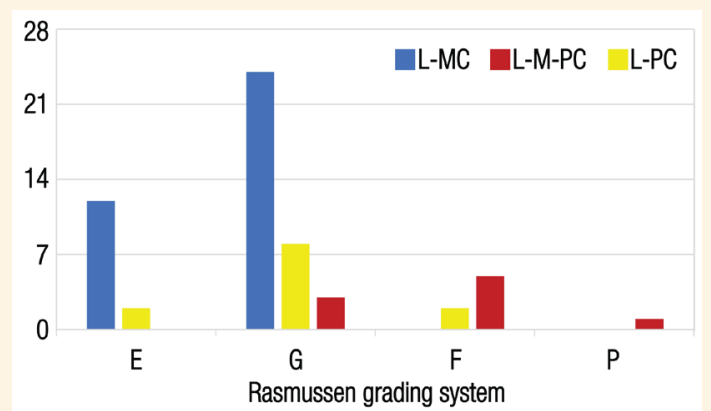

Figure 3: Radiological outcomes of tibial plateau fractures according to the column specific plating.

L-MC:lateral-medialcolumn;L-PC:lateral-posteriorcolumn; $L-M-P C$ : lateral-medial-posterior column; $E=$ excellent; $G$ $=\operatorname{good} ; F=$ fair $; P=$ poor .

= 85-95) for type $\mathrm{V}$ and $87.35 \pm 4.31$ (range $=80-95$ ) for type VI fractures and the WOMAC was 21.64 \pm 7.14 (range $=10.9-29.8)$ and $25.26 \pm 5.24$ (range $=$ 14.9-34.5). Radiological results were classified in four categories as per modified Rasmussen criteria. Of the 57 cases, 14 patients (24.6\%) had excellent results, 35 patients (61.4\%) had good, seven patients (12.3\%) had fair, while only one (1.7\%) had poor results [Table 2]. 

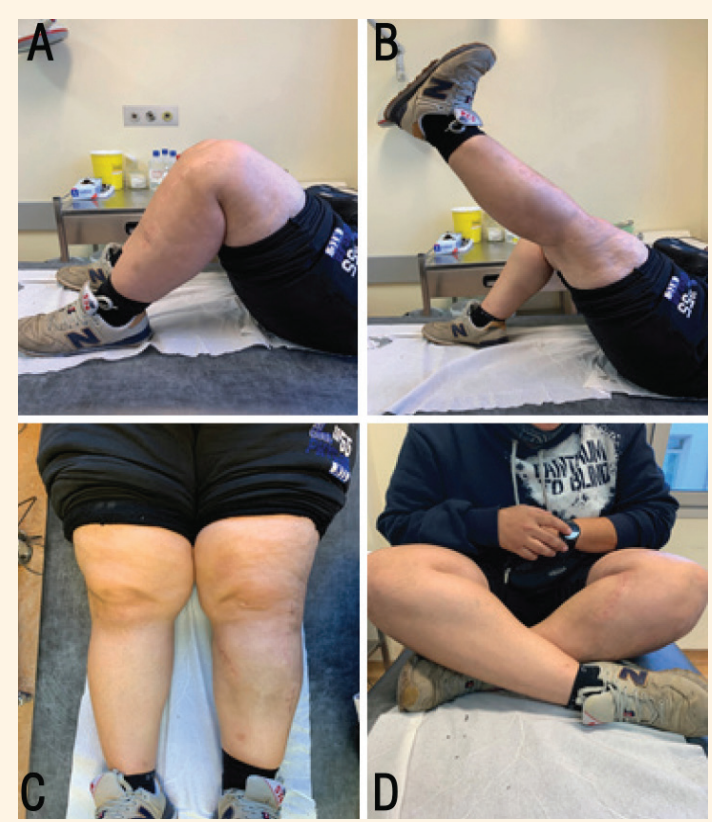

Figure 4: Functional pictures showed good flexion without extension lag (A, B), no varus/valgus malalignment $(\mathbf{C})$ and patient's ability to cross their legs while sitting (D).

Radiological outcomes according to the column specific plating are shown in Figure 3. Statistical analysis showed that there was a significant difference between the Schatzker type V and type VI fractures in VAS and SF-36 $(P<0.05)$, while there was no significant difference on the WOMAC and modified Rasmussen criteria $(P=0.108$ and $P=0.865$, respectively) [Table $2]$. Finally, all the patients were evaluated at the followup for the ROM, the extension lag, the flexion deficit and the malalignment [Figure 4].

\section{Discussion}

Tibial plateau fractures are among the most complex injuries and remain a challenge even for the most experienced orthopaedic trauma surgeons. In order to maintain normal knee joint anatomy and function and accordingly, to ensure the maximal possible ROM, the treatment should be focused on the restoration of joint stability, the alignment and the intraarticular congruity. ${ }^{2}$ ORIF is the gold standard for the management of bicondylar tibial plateau fractures, despite the high rates of compartment syndrome, infection and wound necrosis being reported following placement of dual plating. ${ }^{1-3,9}$

The earlier reports of internal fixation with double plating by Prasad et al. and Pun et al. had shown zero cases of deep tissue infection and only one case with superficial wound infection which healed after hardware removal. ${ }^{10,11}$ Jagdev et al. reported a postoperative superficial infection in $8.69 \%$ of the patients treated with dual plates. ${ }^{3}$ ORIF with double plates in Schatzker type V and type VI fractures had also shown high rates of infections ranging between $11 \%$ and $18 \%{ }^{12-15}$ In the current study, the overall infection rate was $5.3 \%$, which is lower than the one reported in the current literature. It should be noted that the infections in our study were observed only in obese patients. Furthermore, the incidence of compartment syndrome reported in the literature in bicondylar tibial plateau fractures treated with dual plating ranges from $0-13 \% .{ }^{1,10,13,15}$ In the current study, no patient developed compartment syndrome.

Previous studies have demonstrated that the status of the soft tissue envelope at the time of injury and its dissection during surgery has a major impact on the final outcome. ${ }^{3,16}$ Ellsworth et al. have shown that early surgical fixation, within 48 hours of lateral plateau fractures (Schatzker type I to III), can be performed safely and is not associated with a significant increase in the infection or the complication rates. ${ }^{17}$ However, this study found no statistically significant difference in the overall complication and infection rate between fractures (Schatzker type V and VI) treated within 48 hours from the admission and those treated later. This suggests that even complex tibial plateau fractures can be treated immediately with an appropriate preoperative treatment plan. In addition, surgical delay could result in prolonged postoperative hospitalisation and increased overall cost to the healthcare system.

Numerous surgical fixation methods-such as a conventional dual-plate, a hybrid external fixator and/or less invasive stabilizing system-have been proposed for the treatment of complex tibial plateau fractures. ${ }^{7}$ Pun et al. reported a series of 21 bicondylar tibial plateau fractures treated with dual plating or with the Ilizarov fixator. The mean ROM was 128.09 degrees (range $=90-145$ degrees). ${ }^{11}$ Jagdev et al. also reported a series of 26 patients with Schatzker type $\mathrm{V}$ and type VI fractures using conventional dual plating. The mean ROM was 123.4 degrees, while five patients had a functional extension lag of less than 10 degrees. ${ }^{3}$ Christiano et al. demonstrated a mean ROM of 121 degrees following ORIF of bicondylar tibial plateau fractures. ${ }^{15}$ The results of the current study (type $\mathrm{V}=$ 119.35 degrees; type VI = 110 degrees) seem to be quite similar to functional results reported by Christiano et al. ${ }^{15}$ The possible explanations for the differences in the ROM may be due to: i) the surgical technique; ii) the postoperative initiation of physical therapy; and iii) the exact time of the final follow-up.

In 2017, Mengi et al. conducted a retrospective study in 38 patients with type $\mathrm{C} 1$ to $\mathrm{C} 3$ tibial plateau fractures treated with single $(n=23)$ or double plating 
$(\mathrm{n}=15)$ and followed up for at least 12 months. ${ }^{18}$ Clinical outcomes were assessed by the Knee Injury and Osteoarthritis Outcome Score and SF-36 and showed better scores when a single plate was applied relative to a double plate fixation. Pun et al. evaluated clinical and radiological outcomes of 21 patients with Schatzker type V and VI tibial plateau fractures treated with double plates who were followed up for a mean period of 2.5 years. ${ }^{11}$ In that study, the WOMAC questionnaire was used. The authors reported overall satisfactory results without, however, reporting differences between the two treatment groups. The current study also demonstrated favourable clinical outcomes (WOMAC and SF-36) following double plating in both groups, while scores in patients with Schatzker type V fractures were found to be statistically significant compared with patients with Schatzker type VI fractures. The current results are similar to a retrospective study conducted in India. ${ }^{3}$ In that study, the Oxford Knee Score was greater in patients with Schatzker type V fractures treated with dual plating. Furthermore, Hasan et al., in a biomechanical comparative study, suggested that thinner $(3.5 \mathrm{~mm})$ locking plates are likely viable alternatives to $4.5 \mathrm{~mm}$ locking plats and may be associated with less soft-tissue irritation and future wound healing complications. ${ }^{19}$

Soft tissue damage in fractures around the knee joint, limb malalignment and delayed mobilisation are associated with joint instability and may be implicated in the pathogenesis of degenerative knee joint disease. ${ }^{2}$ Parkkinen et al. reported that the articular congruity and restoration of the mechanical axis seem to have a role in prevention of post-traumatic osteoarthritis. ${ }^{20}$ Nevertheless, in a sample of 73 patients, they confirmed that in the mid-term follow-up these factors do not appear to be detrimental to the functional outcomes. The age of the patient at the time of injury seems to be a significant prognostic indicator and correlated negatively with the functional outcomes., ${ }^{3,10}$ In longterm follow-up studies, articular incongruities are well tolerated; conversely, the stability of the knee joint is crucial for the final outcome.,20,21 In the current study, the Rasmussen criteria was used for radiological assessment and a good-to-excellent outcome was achieved in the majority of patients (86\%), which is comparable to other, similar series treated with dual plating. ${ }^{12,22,23}$ According to the same rating system, Yao et al. reported a quite lower radiological result of $77 \%$ good-to-excellent patients after dual buttress plates fixation. ${ }^{22}$ In a series of 34 patients with tibial plateau fracture type V and VI, Rohra et al. reported a result of $94 \%$ good-to-excellent satisfaction rate after plating with dual plates. ${ }^{23}$ Consequently, we suggest that the dual buttress plating technique could achieve favourable fracture reduction and satisfactory clinical outcome.

Tibial plateau fractures are high-energy injuries, commonly associated with ligament, tendon or meniscus injuries, with an overall incidence reported between $12.3 \%$ and $35.2 \%{ }^{2,10,11,24,25}$ The degree of widening of the tibial plateau has been proposed to be a possible predictor of concomitant soft tissue injury. ${ }^{10,11}$ Stevens et al. reported an incidence of ligament injuries in $24 \%$ of the cases of type I to III and $33 \%$ in type IV to VI tibial plateau fractures. ${ }^{24} \mathrm{Yu}$ et al. reported an anterior cruciate ligament injury in $11.1 \%$ of the patients with type V and VI of tibial plateau fractures. ${ }^{25}$ They had also noticed a high incidence of meniscus injury (35.2\%) in their study. In the current study, $21.5 \%$ had soft tissue injuries including ligaments, tendons and meniscus. This marked variation in the incidence of soft tissue injuries reported in the literature may be due to the variety of the methods employed for the diagnosis of these injuries, such as MRI or clinical examination.,210,25

\section{Conclusion}

Despite the relatively small sample size, this study aligns with the results of the current literature. Although technically demanding, ORIF of bicondylar tibial plateau fractures with dual plates is the most stable mechanical construct. The good-to-excellent mid-term radiological (modified Rasmussen criteria) and functional outcomes (SF-36 and WOMAC) support the fact that dual plating of bicondylar tibial plateau fracture is the optimal treatment option. The potential postoperative complications associated with open reduction, such as wound infection and compartment syndrome, can be prevented and/or minimised by general practices including timing of surgical intervention, meticulous soft tissue dissection and suture placement.

\section{CONFLICT OF INTEREST}

The authors declare no conflicts of interest.

\section{FUNDING}

No funding was received for this study.

\section{AUTHORS' CONTRIBUTION}

AVV and DM conceptualised and designed the study. AVV performed the data collection. AVV and FP analyzed the data. AVV and FP drafted the manuscript, while DM and AM revised the manuscript. All authors approved the final version of the manuscript. 


\section{References}

1. Wenger D, Petersson K, Rogmark C. Patient-related outcomes after proximal tibial fractures. Int Orthop 2018; 42:2925-31. https://doi.org/10.1007/s00264-018-3920-0.

2. Khatri K, Lakhotia D, Sharma V, Kiran Kumar GN, Sharma G, Farooque K. Functional evaluation in high energy (Schatzker Type V and Type VI) tibial plateau fractures treated by open reduction and internal fixation. Int Sch Res Notices 2014; 2014:589538. https://doi.org/10.1155/2014/589538.

3. Jagdev SS, Kumar Pathak S, Salunke A, Maheshwari P, Ughareja P, Shah S. Functional outcome of Schatzker type V and VI tibial plateau fractures managed with open reduction internal fixation using dual plates. Int J Res Orthop 2017; 3:961-5. https://doi. org/10.18203/issn.2455-4510.IntJResOrthop20173215.

4. Luo CF, Sun H, Zhang B, Zeng BF. Three-column fixation for complex tibial plateau fractures. J Orthop Trauma 2010; 24:683-92. https://doi.org/10.1097/BOT.0b013e3181d436f3.

5. Lee KW, Yang JS, Hwang JY, Kim KJ, Choy WS. Arthroscopic assessment of cartilage healing status after treatment of tibial plateau fracture. Indian J Orthop 2019; 53:257-62. https://doi. org/10.4103/ortho.IJOrtho_190_17.

6. Chouhan DK, Saini UC, Rajnish RK, Prakash M. Complex bicondylar tibial plateau fractures with reversed tibial slope - Our experience with a fracture-specific correction strategy. Trauma Case Rep 2020; 25:100256. https://doi.org/10.1016/j. tcr.2019.100256.

7. Ryu SM, Yang HS, Shon OJ. Staged treatment of bicondylar tibial plateau fracture (Schatzker type V or VI) using temporary external fixator: Correlation between clinical and radiological outcomes. Knee Surg Relat Res 2018; 30:261-8. https://doi. org/10.5792/ksrr.17.008.

8. The jamovi project (2021). jamovi (Version 1.6) [Computer Software]. From: https://www.jamovi.org Accessed: Oct 2020.

9. Prat-Fabregat S, Camacho-Carrasco P. Treatment strategy for tibial plateau fractures: And update. EFFORT Open Reviews 2016; 1:225-32. https://doi.org/10.1302/2058-5241.1.000031.

10. Thiruvengita Prasad G, Suresh Kumar T, Krishna Kumar R, Murthy GK, Sundaram N. Functional outcome of Schatzker type V and VI tibial plateau fractures treated with dual plates. Indian J Orthop 2013; 47:188-94. https://doi.org/10.4103/00195413.108915.

11. Pun TB, Krishnamoorthy VP, Poonnoose PM, Oommen AT, Korula RJ. Outcome of Schatzker type V and VI tibial plateau fractures. Indian J Orthop 2014; 48:35-41. https://doi.org/10.41 03/0019-5413.125490

12. Zhai Q, Hu C, Lup C. Multi-plate reconstruction for severe bicondylar tibial plateau fractures of young adults. Int Orthop 2014; 38:1031-5. https://doi.org/10.4103/0019-5413.125490.

13. Dall'Oca C, Maluta T, Lavini F, Bondi M, Micheloni GM, Bartolozzi P. Tibial plateau fractures: Compared outcomes between ARIF and ORIF. Strategies Trauma Limb Reconstr 2012; 7:163-75. https://doi.org/10.1007/s11751-012-0148-1.
14. Ozkaya U, Sancar Parmaksizoglou A. Dual locked plating of unstable bicondylar tibial plateau fractures. Injury 2015; 46:S9-13. https://doi.org/10.1016/j.injury.2015.05.025.

15. Christiano AV, Pean CA, Kugelman DN, Konda SR, Egol KA. Function and knee range of motion plateau six months following lateral tibial plateau fractures. J Knee Surg 2020; 33:481-5. https://doi.org/10.1055/s-0039-1678676.

16. Tang X, Liu L, Tu C-Q, Yang T-F, Wang G-L, Fang Y, et al Timing of internal fixation and effect on Schatzker IV-VI tibial plateau fractures. Chin J Traumatol 2012; 15:81-5.

17. Ellsworth HS, Dubin JR, Shaw CM, Alongi SM, Cil A. Immediate versus delayed operative treatment of low-energy tibial plateau fractures. Curr Orthop Pract 2016; 27:351-4. https://doi.org/10.1097/BCO.0000000000000390.

18. Menghi A, Mazzitelli G, Marzetti E, Barberio F, D’Angelo E, Maccauro G. Complex tibial plateau fractures: a retrospective study and proposal of treatment algorithm. Injury 2017; 48:S1-6. https://doi.org/10.1016/S0020-1383(17)30649-6.

19. Hasan S, Ayalon OB, Yoon RS, Sood A, Militano U, Cavanaugh M, Liporace FA. A biomechanical comparison between locked 3.5-mm plates and 4.5-mm plates for the treatment of simple bicondylar tibial plateau fractures: Is bigger necessarily better? J Orthop Traumatol 2014; 15:123-9. https://doi.org/10.1007/s10195-01 3-0275-6.

20. Parkkinen M, Madanat R, Mustonen A, Koskinen SK, Paavola M, Lindahl J. Factors predicting the development of early osteoarthritis following lateral tibial plateau fractures: Mid-term clinical and radiographic outcomes of 73 operatively treated patients. Scand J Surg 2014; 103:256-62. https://doi.org/10.11 $77 / 1457496914520854$

21. Marsh JL, Buckwalter J, Gelberman R, Dirschl D, Olson S, Brown T, et al. Articular fractures: Does an anatomic reduction really change the result? J Bone Joint Surg Amer 2002; 84:1259-71. https://doi.org/10.2106/00004623-200207000-00026.

22. Yao Y, Lv H, Zan J, Li J, Zhu N, Jing J. Functional outcomes of bicondylar tibial plateau fractures treated with dual buttress plates and risk factors: A case series. Injury 2014; 45:1980-4. https://doi.org/10.1016/j.injury.2014.10.004.

23. Rohra N, Singh Suri H, Gangrade K. Functional and radiological outcome of Schatzker type V and VI tibial plateau fracture treatment with dual plates with minimum 3 years follow-up: A prospective study. J Clin Diagn Res 2016; 10:RC05-RC10. https://doi.org/10.7860/JCDR/2016/18732.7855.

24. Stevens DG, Beharry P, McKee MD, Waddell JP, Schemitsch EH. The long-term functional outcome of operative treated tibial plateau fractures. J Orthop Trauma 2001; 15:312-20. https:// doi.org/10.1097/00005131-200106000-00002.

25. Yu Z, Zheng L, Zhang Y, Li J, Ma B. Functional and radiological evaluations of high-energy tibial plateau fractures treated with double-buttress plate fixation. Eur J Med Res 2009; 14:200-5. https://doi.org/10.1186/2047-783x-14-5-200 